\title{
Erratum
}

\section{Individual and Social Learning}

\section{NOBUYUKI HANAKI}

Doctral Program in International Political Economy, Graduate School of Humanities and Social Sciences, University of Tsukuba, 1-1-1 Tennodai, Tsukuba, Ibaraki, 305-8573, Japan E-mail: hanaki@dpipe.tsukuba.ac.jp

Accepted 21 July 2004

Unfortunately the page numbers online for this article are incorrect. The correct page numbers should be: 213-232. 